Vol. 48 (1993) [171-172]

\title{
Varieties of quasigroups and related topics
}

\section{DARRYN E. BRYANT}

It is possible to obtain various algebras by defining binary operations on the underlying sets of certain types of combinatorial designs. Conversely, combinatorial designs may be constructed from, and classified by, their corresponding algebras.

Many of the combinatorial designs dealt with can be described in (at least) two equivalent ways, either as block designs with a cyclic order attached to each block or as a graph decompositions. Two of the well known combinatorial designs discussed are cycle systems and Mendelsohn designs. Quasigroups are the main class of algebras examined.

Chapter I contains the preliminary definitions and results that are used in later chapters. It is divided into four sections, Universal algebraic preliminaries, Some examples of varieties, Combinatorial design preliminaries and Graph theoretic preliminaries.

In Chapter II, L-circuit systems are introduced and their one-one correspondence with a variety of algebras is examined, see [3]. All of the subsequent classes of combinatorial designs examined may be considered subclasses of this general class and the correspondence (with slight adaptations) is used throughout. The major subclasses are described in the remaining sections of this chapter.

Chapter III is a detailed look at one of the classes of algebras, namely $P$-quasigroups, that appeared in Chapter II. It contains several results concerning homomorphisms and varieties of $\mathrm{P}$-quasigroups, see $[1,2,4]$. One of these results provides, as a corollary, a solution to a problem concerning the existence of identities that characterise quasigroups arising from 2-perfect 6 -cycle systems, see $[6,7]$.

Chapter IV introduces a method of constructing identities which hold in any given finite quasigroup, see [5]. The method is based on repeated application of a process to a variable until an identity is produced and it gives rise to identities called repetitive operation laws or rols. These rols are used to provide results which answer questions arising out of previous chapters.

In contrast to Chapter IV which deals with algebraic questions arising out of combinatorics, Chapter V looks at existence questions concerning combinatorial designs which

Received 22nd March, 1993

Thesis submitted to The University of Queensland, November 1992. Degree approved, March 1993. Supervisor: Dr S. Williams

Copyright Clearance Centre, Inc. Serial-fee code: 0004-9729/93 \$A2.00+0.00. 
to some degree owe their origins to universal algebra. The existence results obtained for the designs constructed in Chapter $V$ may also be described in graph theoretical terms. Specifically, necessary and sufficient conditions for a decomposition of the complete directed looped graph of order $n$ into closed trails of length $m$ are given, see [3]. The harder problem of existence of such decompositions whose corresponding algebras are quasigroups is also examined.

Chapter VI contains various miscellaneous results concerning a special class of combinatorial designs called Steiner systems.

\section{REFERENCES}

[1] D.E. Bryant, 'Varieties of quasigroups arising from 2-perfect $m$-cycle systems', Des. Codes Cryptogr. 2 (1992), 159-168.

[2] D.E. Bryant, 'Varieties of P-quasigroups', Australas. J. Combin. 6 (1992), 229-243.

[3] D.E. Bryant, 'Decompositions of directed graphs with loops and related algebras', Ars Combin. (to appear).

[4] D.E. Bryant and C.C. Lindner, '2-perfect $m$-cycle systems can be equationally defined for $m=3,5$ and 7 only', Algebra Universalis (to appear).

[5] D.E. Bryant and S. Oates-Williams, 'Constructing laws for finite quasigroups', Comm. Algebra (submitted).

[6] C.C. Lindner, 'Graph decompositions and quasigroup identities', in Proceedings of the 2nd International Catania Combinatorial Conference, Graphs, designs and combinatorial geometrics (Universita di Catania, Sicily, September 4-9, 1989). Le Matematiche XLV (1990), 83-118.

[7] C.C. Lindner, K.T. Phelps and C.A. Rodger, 'The spectrum for 2-perfect 6-cycle systems', J. Combin. Theory Ser. A 57 (1991), 76-85. 